\title{
The Potential of Liquid Marbles for Biomedical Applications: A Critical Review
}

\author{
Nuno M. Oliveira, Rui L. Reis, and João F. Mano*
}

Liquid marbles (LM) are freestanding droplets covered by micro/nanoparticles with hydrophobic/hydrophilic properties, which can be manipulated as a soft solid. The phenomenon that generates these soft structures is regarded as a different method to generate a superhydrophobic behavior in the liquid/ solid interface without modifying the surface. Several applications for the LM have been reported in very different fields, however the developments for biomedical applications are very recent. At first, the LM properties are reviewed, namely shell structure, LM shape, evaporation, floatability and robustness. The different strategies for LM manipulation are also described, which make use of magnetic, electrostatic and gravitational forces, ultraviolet and infrared radiation, and approaches that induce LM self-propulsion. Then, very distinctive applications for LM in the biomedical field are presented, namely for diagnostic assays, cell culture, drug screening and cryopreservation of mammalian cells. Finally, a critical outlook about the unexplored potential of LM for biomedical applications is presented, suggesting possible advances on this emergent scientific area.

\section{Introduction}

Liquid marbles (LM) consist in liquid droplets coated with a hydrophobic powder, according Aussillous and Quéré that reported for the first time this concept. ${ }^{[1]} \mathrm{LM}$ production is achieved by simply rolling a liquid droplet over hydrophobic micro or nanoparticles. Where the encapsulated material is protected by the hydrophobic shell, preventing the physical contact between the liquid and the external environment. ${ }^{[1-4]}$ However,

\author{
N. M. Oliveira, Prof. R. L. Reis, Prof. J. F. Mano ${ }^{[+]}$ \\ 3B's Research Group - Biomaterials \\ Biodegradables and Biomimetics \\ University of Minho \\ Headquarters of the European Institute of Excellence on Tissue \\ Engineering and Regenerative Medicine \\ AvePark, Zona Industrial da Gandra \\ 4805-017 Barco GMR, Portugal \\ E-mail: jmano@ua.pt \\ N. M. Oliveira, Prof. R. L. Reis, Prof. J. F. Mano \\ ICVS/3B's - PT Government Associate Laboratory \\ Braga/Guimarães, Portugal \\ ${ }^{[+]}$Current address: Department of Chemistry, CICECO - Aveiro Institute \\ of Materials, University of Aveiro, Aveiro, Portugal
}

The ORCID identification number(s) for the author(s) of this article can be found under https://doi.org/10.1002/adhm.201700192.

DOI: $10.1002 /$ adhm.201700192 it was also reported that hydrophilic particles can be used on LM production, specifically graphite and carbon black. ${ }^{[5,6]} \mathrm{LM}$ make easier the manipulation of liquids which combined with other intrinsic properties justify the growing interest of the scientific community.

Widely range of applications were found for LM, such as oil adsorption and separation, ${ }^{[7,8]} \mathrm{pH}$ and gas sensing, ${ }^{[9-13]}$ chemical reactions, ${ }^{[14,15]}$ synthesizing microparticles, ${ }^{[16]}$ revealing water pollution on the water/vapor interface ${ }^{[17]}$ and manipulation of small amounts of liquids (micro-reactors, micro-pumps). ${ }^{[14,18,19]}$ The LM use in cosmetics has been also suggested, due to the non-oily feel imparted to skin by marbles. ${ }^{[20]}$ Several studies and applications for LM have been published in different scientific fields, but only very recently biomedical applications have been reported.

Some excellent reviews targeting LM topic exist in literature. The scopes of these reviews cover general properties, manipulation and applications of LM. ${ }^{[2-4,21-25]}$ In contrast, the aim of the review presented here was to focus on the emerging field of biomedical applications for LM. Based on the most recent progress, we intended to show how the properties and manipulation of LM were explored to develop the reported biomedical applications. This review is finalized with a critical view of the latent potential of LM for new applications on the referred field. Therefore, this paper is composed by three sections: a) compilation of LM properties and developed methodologies for LM manipulation, b) illustration of the most recent progress in the biomedical applications for LM, and c) discussion of the LM potential for new biomedical applications based on the recent progress.

\section{Liquid Marbles}

\subsection{Properties}

\subsubsection{Shell Structure}

LM are non-stick droplets covered by micro and/or nanometrically scale particles with hydrophobic (and few hydrophilic) properties which enable to manipulate the marbles like a soft solid - Figure 1A. This soft solid is regarded as an alternative 
approach to superhydrophobicity, once also inhibits the wetting of liquid droplets on solid surfaces. The original superhydrophobicity concept postulated that the anti-wetting properties were achieved by physical and/or chemical modification of a solid surface. ${ }^{[26-28]}$ In the case of LM the approach is opposite, the modification is performed on the liquid droplet interface with the external environment instead of the supporting surface. Due to the non-wetting/non-sticking property, LM present very low friction with a wide diversity of solid and liquid supports. This low-friction characteristic allows LM to move easily and reveals to be crucial on both manipulation and development of numerous LM applications..$^{[14,24,29,30]}$

Hydrophobic particles can be loosely fixed over flat surfaces to prepare moldable superhydrophobic surfaces. ${ }^{[31]}$ For the case of LM, it was reported that the encapsulating layer of particles can present a structure from a loosely packed monolayer to a multilayer structure. Generally, LM coating has a non-uniform thickness, consisting in an arrangement of mono- and multilayers of particles - Figure 1B. ${ }^{[32,33]}$ Morphologically, LM wall presents significant roughness and porosity in appearance and is composed by particle aggregates separated by liquid region spacing (liquid clearings) - Figure 1B. ${ }^{[33-35]}$ Due to the roughness and the porosity, air remains entrapped on LM shell creating "air pockets", providing an analogous outcome to the "lotus effect" on rough surfaces..$^{[3,36]}$ The air pockets give also to the LM the interesting ability to float in liquids Figure $1 \mathrm{~A} .{ }^{[30,34,37]}$ The absence of direct contact between the encapsulated fluid and both solid and liquid supports was experimentally confirmed. ${ }^{[34]}$ However, it was clearly demonstrated that the powder shell coating LM is permeable for gases, allowing their use in gas sensing and biological applications (reviewed ahead). ${ }^{[12,13,38-41]}$

\subsubsection{Liquid Marble Shape: Static, Rolling and Floating Conditions}

The shape of LM is determined by the equilibrium between the effects of gravity and surface tension. ${ }^{[2,3]}$ As marbles are soft, they deform in a way to minimize their potential energy. Increasing their size, LM tend to lower their center of mass and consequently increase the contact area with the supporting surface. ${ }^{[43]}$ Wherein, small LM present a quasi-spherical shape, due to the negligible effect of gravity. On the other hand, in large LM the gravity force predominates on the shape formation, originating marbles with flattened puddle shapes. ${ }^{[1,33]}$ The classification of small and large is performed by comparison to the capillary length, classifying as small marbles the ones that present a height significantly less than the double of the capillary length. ${ }^{4,21]}$

Due to the very low friction between the LM and the supporting surface, LM can easily move down a tilted substrate. ${ }^{[1]}$ Here, LM do not slide but roll, and larger marbles roll more slowly comparing with smaller LM. ${ }^{[43,44]}$ Moreover, it was shown that rotating marbles can deform into doughnut and peanut shapes, due to the centrifugal force influence combined with LM coating flexibility - Figure $2 .^{[1,2]}$

Recently, Ooi et al. suggested a model to characterize the deformation of LM floating in a liquid surface. ${ }^{[45]}$ A floating oblate spheroid model was developed from the floating solid

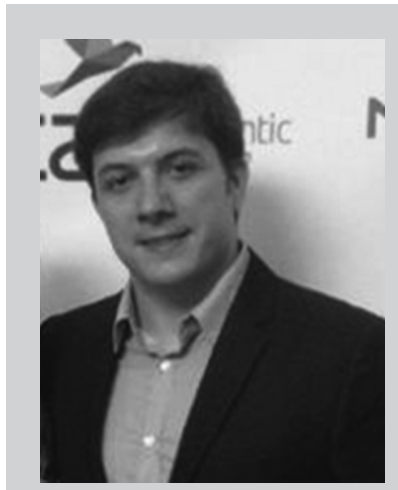

Nuno M. Oliveira (MSc) is a student of the Doctoral Program on Tissue Engineering, Regenerative Medicine and Stem Cells (TERM\&SC) at University of Minho. He completed his Master degree in Biological Engineering at the same university. He has been working on the development of biomedical devices engineered based on the advanced control of the surface wettability. In 2015, he had the opportunity to integrate the BIRC team at Harvard Medical School (USA), working on the design and production of innovative floating hydrogels.

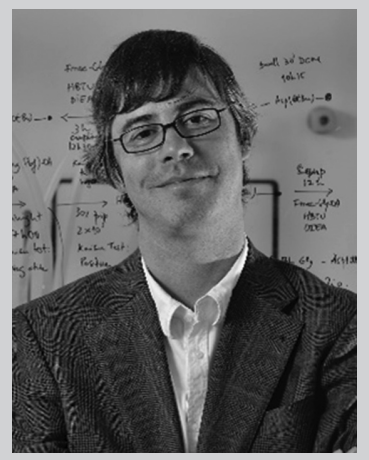

Rui L. Reis (PhD, DSc, Hon. Causa MD, FBSE, FTERM, member of NAE) is the Vice-President for R\&D of University of Minho, Portugal, Director of the 3B's Research Group and of the ICVS/3B's Associate Laboratory of UMinho. $\mathrm{He}$ is also the CEO of the European Institute of Excellence on Tissue Engineering and Regenerative Medicine, the Global President of the Tissue Engineering and Regenerative Medicine International Society (TERMIS) $\mathrm{He}$ is a recognized World expert in the development of biomaterials from natural origin polymers and their use in tissue engineering and regenerative medicine, and in controlled drug delivery, in many cases in combination with different types of stem cells.

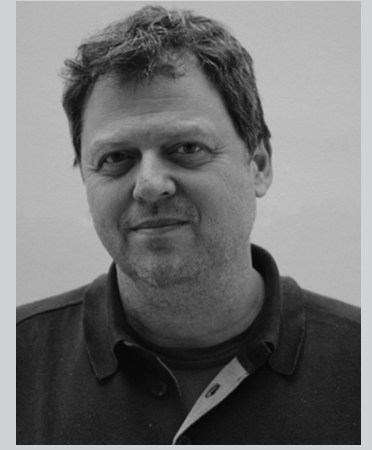

João F. Mano (CEng, PhD, DSc) is a Full Professor at the Chemistry Department of University of Aveiro. His current research interests include the use of biomaterials and cells cells towards the progress of transdisciplinary concepts to be employed in regenerative and personalised medicine. In particular, he has been applying biomimetic and nano/micro-technology approaches to polymer-based biomaterials and surfaces in order to develop biomedical devices with improved structural and (multi-)functional properties, or in the engineering of microenvironments to control cell behaviour and organization, to be exploited clinically in advanced therapies or in drug screening. 

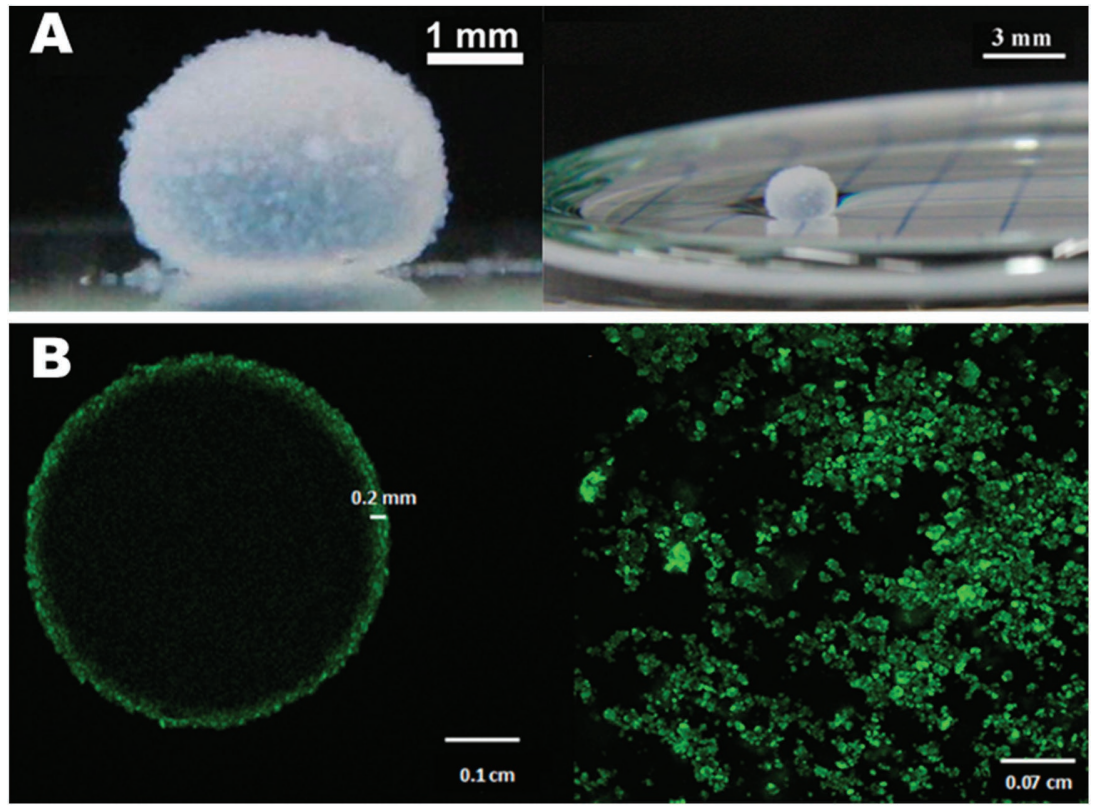

Figure 1. A) A LM resting on a glass slide and floating on the surface of glycerol. Reproduced with permission. ${ }^{[42]}$ Copyright 2015, American Chemical Society. B) Image of a LM and view of a cross section of the LM coated with a fluorescein O-methacrylate based powder. Reproduced with permission. ${ }^{[33]}$ Copyright 2015, American Chemical Society.

sphere model. ${ }^{[46]}$ Additionally, they reported that the developed model is different than the model proposed by Whyman and Bormashenko, for a sessile droplet resting on a solid surface. ${ }^{[47]}$ This was justified by the fact that for the floating LM case a deformation occurs in both the marble and the liquid surface. These models may be important for improving the models of LM evaporation, since the exposed surface area to air may change for the different cases.

\subsubsection{Evaporation Phenomenon}

The evaporation of LM is one of the most important parameters for long term applications (e.g. biomedical applications), because with the evaporation LM loose their shape and collapse. ${ }^{[23,24,48]}$ Several works have studied the evaporation phenomenon in LM, analyzing the effect of different types of coating particles and layer organizations. ${ }^{[5,49-55]}$ The encapsulated liquid is also important and, as expected, for more volatile liquids the evaporation rate is higher. ${ }^{[55]}$

Dandan and Erbil reported that graphite-coated LM presented lower evaporation rates comparing with bare water
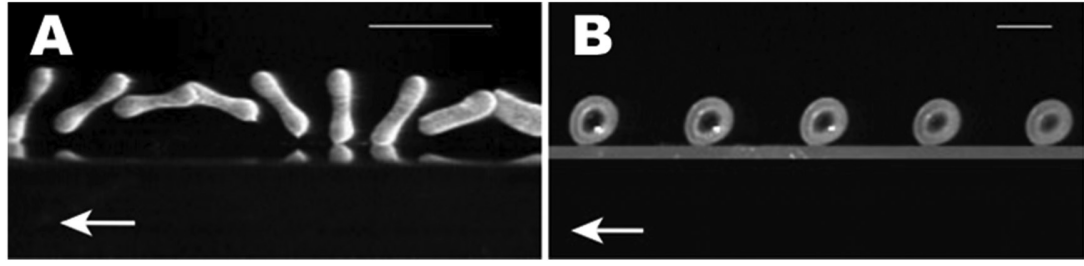

Figure 2. Different shapes taken by a liquid marble in the inertial regime. A) Peanut and B) doughnut shapes of a rotating marble. Arrow indicates direction of the motion; scale bar: $1 \mathrm{~cm}$. Reproduced with permission. ${ }^{[1]}$ Copyright 2001, Macmillan Publishers Ltd. droplets in the same conditions, presenting the LM a lifetime twice longer than the water droplets. ${ }^{[5]}$ However using hydrophobic polytetrafluoroethylene microparticles ( $\mu$ PTFE) on the water-air interface of LM, the water marbles just presented 5 to $35 \%$ more of lifetime than of the pure water droplets. ${ }^{[50]}$ Bhosale et al. compared $\mu$ PTFE with fumed silica nanoparticles chemically modified with two different hydrophobic molecules: hexamethyldisilazane and dimethyldichlorosilane. ${ }^{[51]}$ The obtained results showed that the water diffusion through LM shell was not related with the particle size. Instead, the authors suggested that the higher resistance to water loss of LM coated with hexamethyldisilazane-modified nanoparticles may be attributed to the lower aggregation of this particles. Recently, Laborie et al. also suggested that the rate of water evaporation is independent of the particle size. ${ }^{[53]}$ However, unlike Bhosale et al., they associated the slower drying of LM with the presence of multilayer coatings and they suggested that the multilayers may be formed by aggregates of nanoparticles. This work indicated that LM covered with a monolayer of hydrophobic particles dried faster than bare liquid droplets. On the other hand, multilayer coated LM dried slower than water droplets. Additionally, it was found that the evaporation rate of LM is directly related with the ratio between shell thickness and LM diameter. ${ }^{[53]}$ Ooi et al. also suggested that the coating density could be an important factor driving the liquid evaporation in LM..$^{[55]}$

Despite the several studies on this subject, more studies seems necessary to fully understand the relationship between the evaporation behavior in LM and the coating structure.

\section{1.4. Floating Ability}

In literature several works studying and exploring the ability of LM to float can be found - Figure 1A. ${ }^{[9,11,17,34,37,42,45,50,56-63]}$ Taking advantage from this LM property, applications were developed on sensors field for $\mathrm{pH}$-sensing, ${ }^{[9-11,57-59]}$ to reveal water pollution, ${ }^{[17]}$ and also on development of floating selfpropelling devices. ${ }^{[42,60]}$ Generally, $\mathrm{pH}$-sensing LM were coated with $\mathrm{pH}$-responsive particles that change their wettability from hydrophobic to hydrophilic with $\mathrm{pH}$ variations. These LM usually exhibit long-term stability floating in solutions with a $\mathrm{pH}$ above or below a defined value. With the addition of an acid ${ }^{[57,58]}$ or a base ${ }^{[9,11,59,63]}$ to the supporting liquid, the LM immediately disintegrate due to the increase of the coating hydrophilicity. The $\mathrm{pH}$ changes can be visually detected by LM destruction and/or by colorant release. For the detection of water pollution the mechanism is similar, but instead of a change on LM shell the alteration occurs on the supporting liquid. 
As an example of this application, LM were able to successfully recognize the water contamination by organic compounds. ${ }^{[17]}$ With these contaminants, the water surface tension decreased and the floating LM lost their integrity, revealing the water contamination without water sampling. On the same topic of responsive floating LM to external stimuli, Nakai et al. reported a thermoresponsive LM that lost their integrity when the temperature increased and reached $46.5{ }^{\circ} \mathrm{C} .{ }^{[64]}$ This temperature corresponded to the melting point of the material used in the LM coating. The authors suggested that these marbles can be used for chemical reactions between the LM interior and the supporting liquid, being the reaction triggered by heating.

\subsubsection{Effective Surface Tension}

Encapsulating a liquid with nano/microparticles, the surface tension of the pure liquid is altered to form the marble. The corresponding surface tension of the LM is defined as the "effective surface tension", which is the specific energy of the liquid coated with the particle/vapor interface. ${ }^{[22,37]}$

Essentially, five different techniques have been suggested for the establishment of the effective surface tension of LM: i) the pendant marble method; ii) methods using the vibration of marbles; iii) capillary rise and Wilhelmy plate methods; iv) analysis of the LM shape; and, v) the puddle height method that is the most popular and simple method. There are two papers that summarize and explain all of these different methods: one is from Bormashenko ${ }^{[3]}$ and other from Cengiz and Erbil. ${ }^{[3]}$ However, the current experimental situation is complex and many different values have been reported for the effective surface tension, because physical properties of surfaces stabilized with solid particles depend on the density and nature (hydrophobicity of the powder, particle size) of shell. ${ }^{[3,22,37]}$ Thus, more experimental studies are claimed to clarify this situation and understand the influence of the physical nature and density of coating particles on the surface properties of LM.

\subsubsection{Robustness/Elasticity}

Understanding the mechanical robustness of LM is very important to design applications that involve transportation and manipulation of LM. This robustness comprises the capability of marbles to resist to deformation, pressure and impact.

In static conditions, the resistance to deformation and pressure could be assessed by quasi-static compression methods, showing the LM high elasticity - Figure 3. ${ }^{[65,66]}$ For small mechanical compression, Asare-Asher et al. reported that LM could sustain a purely elastic deformation of up to $30 \%$ of their original size, using potassium chloride solution encapsulated by polyethylene microparticles $(53-75 \mu \mathrm{m})$ - Figure 3.[66] The elastic properties of LM are attributed to two very distinct mechanisms. ${ }^{[66,67]}$ Thus, one mechanism is the interfacial force, namely the capillary interactions between the colloidal particles coating the marbles. ${ }^{68,69]}$ The other mechanism is common to LM and droplets, which is the restoring "spring-like" force driven by the surface tension that permits to LM recover their
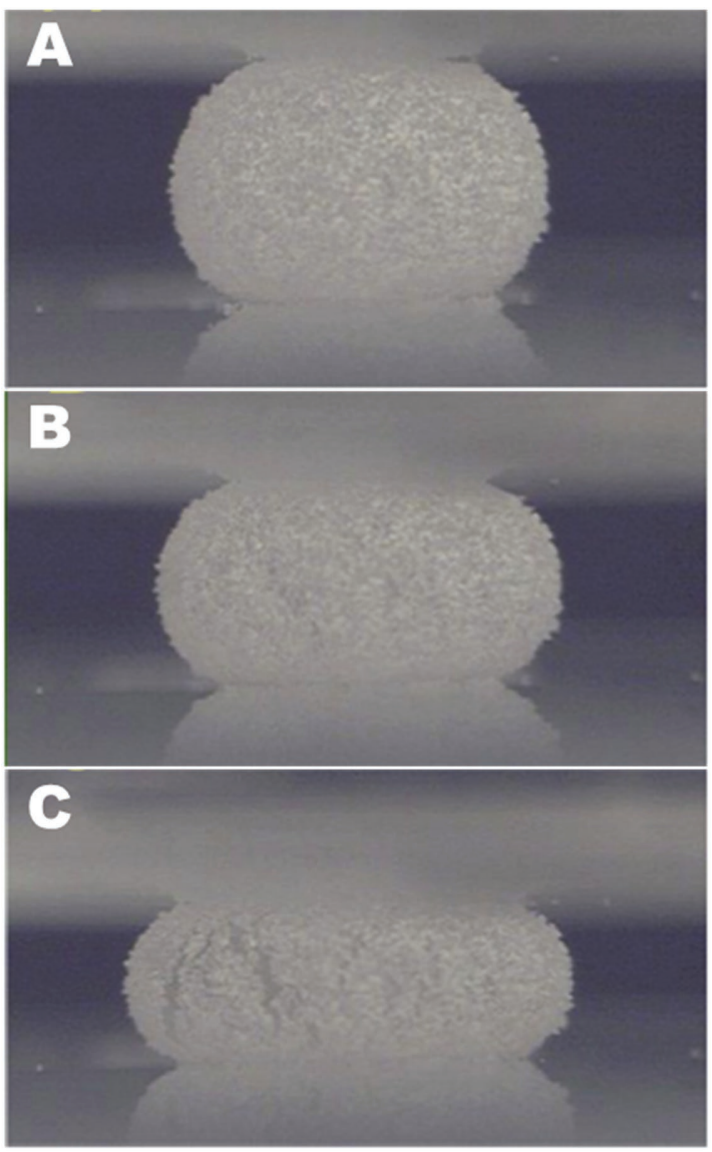

Figure 3. Liquid marble under compression: A) Small deformation; B) medium deformation - cracks in the particle coating can be seen under scrutiny; and C) breakage point - immediately before LM rupture. Reproduced with permission. ${ }^{[66]}$ Copyright 2015, Elsevier.

shape after deformation. ${ }^{[0,71]}$ This same elastic behavior was found with LM under compression conditions and during the noncoalescent collisions of LM. ${ }^{[68,69]}$ Using high mechanical compression, Liu et al. studied the critical pressure before LM rupture, attributing this rupture to the poor coverage of particles on marble surface when highly stretched. ${ }^{[72]}$ They found that the mechanical robustness of marbles was influenced by the hydrophobicity and size of the coating particles.

Regarding to particles hydrophobicity, Liu et al. found that fumed silica particles with a apparent water contact angle (AWCA) of $118^{\circ}$ created more robust LM comparing with particles presenting $145^{\circ}$ - AWCA was measured by placing a water droplet over glass slides spin coated with a layer of fumed silica particles. ${ }^{[72]}$ They attributed this result to the fact that the AWCA of particles was closer to $90^{\circ}$, because at this AWCA particles are more strongly bound to the liquid surface. ${ }^{[4,72]}$ This explanation was corroborated from other works that experimentally demonstrated higher mechanical robustness for a layer at the liquid-air interface using particles with an AWCA of $90^{\circ}{ }^{[73]}$ Zang et al. also confirmed this results, showing that a coating using particles with properties in limit of the hydrophobic to hydrophilic regime presented the highest mechanical robustness for LM. ${ }^{[7]}$ For this study, silica-based nanoparticles $(\approx 20 \mathrm{~nm})$ with different relative $\mathrm{SiOH}$ content and an AWCA 
in the range of $80-135^{\circ}$ were used. ${ }^{[73-75]}$ However, Zhou et al. found higher mechanical stability for the LM produced using particles with an AWCA of $132^{\circ}$, from a studied AWCA range of $84-132^{\circ} .^{[76]}$ For the coating, acetylated cellulose powders (average size of $\approx 40 \mu \mathrm{m}$ ) with different degrees of acetylation were used. This unexpected result may be owing to the very irregular shape of the coating powders. ${ }^{[76]}$ Once the theoretical explanation for the AWCA of $90^{\circ}$ such as the best for the highest LM robustness is based on particles with smooth and spherical shape, ${ }^{[4]}$ this explanation may do not fit in the specific case of Zhou et al. More meticulous studies aiming this subject are indispensable.

As previously mentioned, it was reported that the particle size also plays an important role regarding to marble robustness. Several reports stated that smaller particles generate more stable LM. ${ }^{[3,37,51,72]}$ It has been found that nanoparticles behave like an "elastic membrane", creating very robust marbles in comparison with microparticle coatings. ${ }^{[1,72]} \mathrm{A}$ possible explanation is that increasing the particles size also increase the liquid clearings on the layer of particles, weakening the LM shell. ${ }^{[3-35,72]}$ Using nanoparticles, the shell is strengthened due to a more uniform deposition and a better network created between nanoparticles based on the attraction imposed by lateral capillary forces. ${ }^{[51,72,77]}$

The mechanical robustness of LM surprisingly allow procedures such as inject and extract liquid from the LM core without destroy them. ${ }^{[48,78,79]}$ Bajwa et al. reported a deeply study to characterize the behavior and the operational limits of LM upon liquid exchange via external inflow and outflow. ${ }^{[78]}$ They reported that with the liquid injection a swirl motion on the marble surface occurred. Increasing injection flow rate the shear forces increased, consequently also the swirl motion and at high swirl rates LM robustness decreased. However, for moderate levels of swirl an enhanced on the robustness was observed. Larger marbles showed to take more liquid before disruption comparing with smaller marbles. It was also reported that the liquid can be removed and re-injected from the LM core and the marbles regained their spherical shape and non-wetting behavior. With these extractioninjection cycles, the LM were weakening and disrupted after three cycles. ${ }^{[78]}$ Finally, the authors showed be possible simultaneously inject and extract the liquid from the LM, being possible to use the marbles for perfusion experiments at low flow rates. Thus, LM present high potential as bioreactors for longterm cellular culture, since the cell culture medium can be exchanged continuously by perfusion. For this application, low flow rates are important to avoid cell wash-out from reactor and mimic in vivo conditions.

Sun et al. reported that LM can be manipulated using tweezers. ${ }^{[80]}$ The mechanical integrity of LM stabilized by charged polystyrene-latex particles showed the possible use of tweezers to completely cut marbles without destroy them. Their shape became elongated by mechanical forces but can be restored again, always using the tweezers.

\subsection{Liquid Marbles Manipulation}

Due to transforming a liquid-solid interaction into a solid-solid interface, LM are non-stick droplets presenting an extremely low friction with the supporting surface, and consequently small forces for LM manipulation are required. Several different manipulation strategies have been reported, namely using magnetic, electrostatic and gravitational forces. Recently, the use of ultraviolet, infrared irradiation and self-propelling marbles were also reported.

\subsubsection{Magnetic}

One of the most common means for manipulation of LM is the use of magnetic forces. Its popularity is owed to the simplicity of use and the remote control (no physical contact required) Figure 4A. $[7,14,24,25,29,30,81-88]$ There is two means to confer

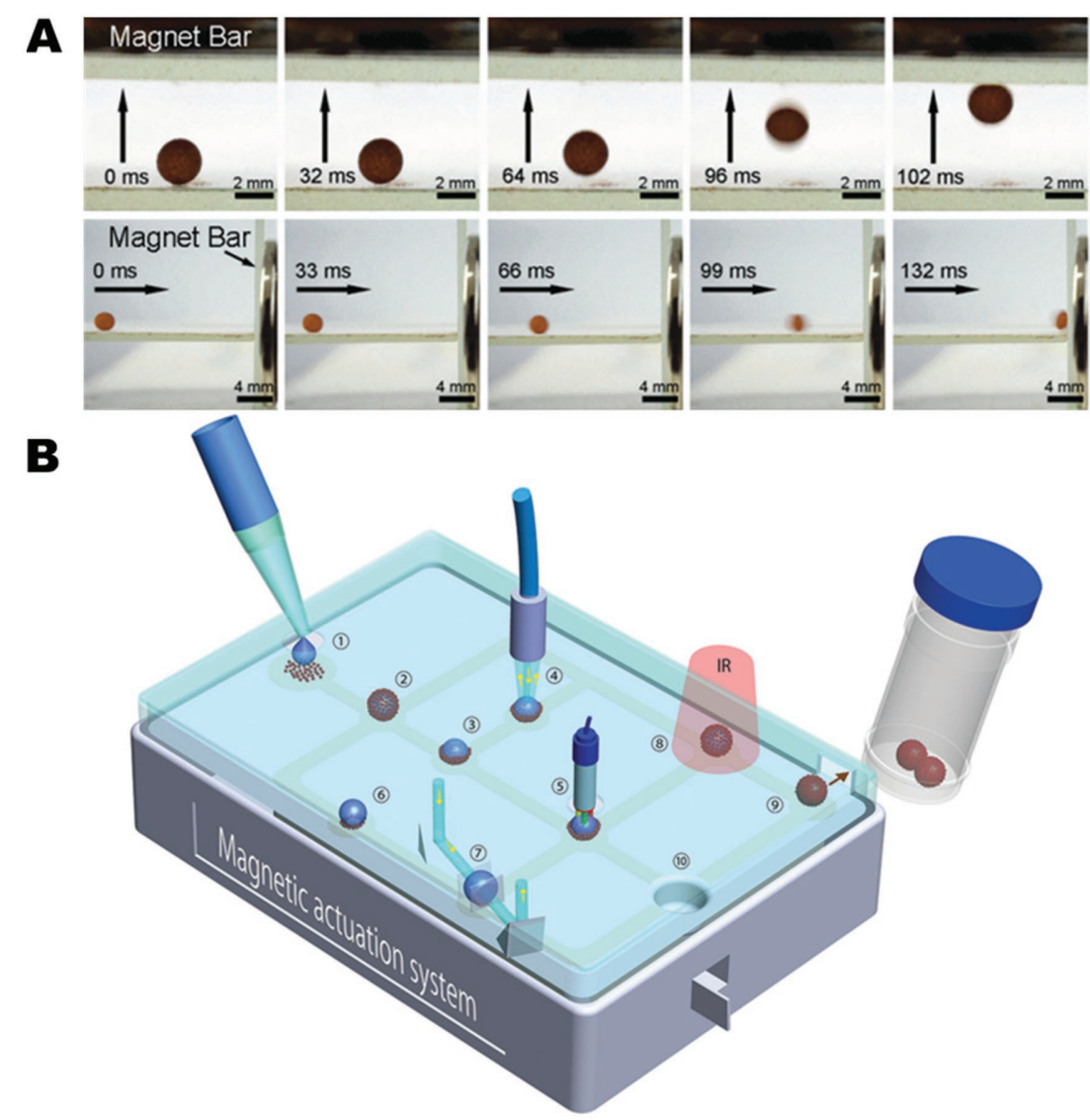

Figure 4. A) Images of a LM moving vertically and horizontally driven by a magnet bar. Reproduced with permission. ${ }^{88]}$ Copyright 2016, the Royal Society of Chemistry. B) Illustration of a proposed device for "on-line" detection and for sample encapsulation based on magnetic LM. Reproduced with permission. ${ }^{[85]}$ 
magnetic response in LM: a magnetic coating ${ }^{[84,87,88]}$ or a magnetic content. ${ }^{[81,86]}$

For the coating, the used particles are hydrophobic nanoparticles of iron $\left(\mathrm{Fe}_{3} \mathrm{O}_{4}\right)^{[29,84]}$ or synthesized nano/microparticles combining iron and other materials..$^{[7,87,88]}$ Some of these synthesized microparticles were produced using biocompatible materials which opens the possibility to use them in biomedical applications, being an example of the magnetic superhydrophobic cellulose-based microspheres developed by Lin et al. ${ }^{[88]}$ Fundamentally, the iron is used to confer superparamagnetic properties to the coating particles, making possible the LM manipulation by using an external magnetic field - Figure 4A. Since the hydrophobic coating powder can be manipulated, these magnetic marbles allowed the hydrophobic coating to open and to close reversibly and also the controllable merging of two marbles. ${ }^{[14,29]}$ Marbles with magnetic properties roll after a moving permanent magnet and do not slide on the solid surface, as the regular LM. ${ }^{[14]}$

By taking advantage of the LM feature of being opened and closed reversibly, it was possible to have directly access and add a second liquid to the marble core, producing a bicomponent LM suitable for chemical reactions. ${ }^{[14]}$ This LM feature also allowed to perform optical detection with a reflection mode that enabled to probe chemical reactions taking place within marbles. ${ }^{[83]}$ By exploring the easy manipulation of magnetic LM, Zhao et al. proposed to integrate different processes in a single device - Figure 4B. Using magnetism, the LM could be moved along of the proposed device and the marbles could be opened to perform electrochemical measurements, introducing an electrode probe inside of LM and also through optical detection. ${ }^{[85]}$ Recently, it was also proposed a device to magnetically actuate in floating LM, aiming the transport of aqueous solution with minimal volume loss using a digital microfluidics platform. ${ }^{[86]}$ The authors proposed that the platform may be used in biological applications, namely as a bioreactor for growing cells. Here, the magnetic properties of LM were provided by the iron oxide particles added to the marbles content. These magnetic particles showed to be biocompatible, however their biocompatibility can be improved by coating the particles with surfactants such as polyethylene glycol or sodium oleate. ${ }^{[86,89]}$

\subsubsection{Electrostatic}

It was showed that LM can also be deformed, moved and can experience changes in their wettability when exposed to an electric field. ${ }^{[2,90-96]}$ Aussillous and Quéré showed for the first time that LM can be moved by the effect of an electrostatic field, where this field was reached by simply rubbing a stick of Teflon on a fabric ${ }^{[2]}$ By using electrostatic interactions, it was also showed that a liquid droplet can be spontaneously coated with hydrophobic particles, resulting in multi-layered LM. ${ }^{[92]}$ By exposing LM to an electric field provided by a capacitor, LM could be actively deformed ${ }^{[93]}$ and controlled their movements. ${ }^{[94,95]}$ Similar behavior was also reported for "Janus" marbles, which are droplets composed of two hemispheres characterized by presenting different physical and/or chemical properties. It was showed that "Janus" marbles could be rotated with an electric field due to the difference in electric properties of the particles used for the coating, in this case Teflon and carbon black that are dielectric and semiconductor particles respectively. ${ }^{[96]}$

However, there is a lack of studies showing the safety of electric fields used in LM manipulation for biomedical applications, namely the effect of electric fields in mammalian cells needs to be assessed.

\subsubsection{Gravitational}

The simplest ways to provide movement to LM is allowing it to roll down an inclined plane, during which gravitational potential energy is transformed into kinetic energy conducting to a descending movement. ${ }^{[24,97]}$ The speed acquired by the LM is influenced by the inclination angle, surface tension, viscosity and Bond number. ${ }^{[2,43]}$ Smaller marbles roll faster comparing with larger LM. ${ }^{[43,44]}$ Two marbles can be merged in a controlled way under the action of gravity. ${ }^{[98,99]}$ Based on this method, Castro el al. presented a 3D printed platform for continuous production of "Janus" and composite LM. ${ }^{[99]}$ The marbles were produced through the coalescence of two LM with different coatings and the process control was performed by adjusting the inclination angle of the designed platform. The authors suggested that the developed device has potential application for surface engineering with innovative functionality for drug therapies, particles-based barcode biomarkers and smart membranes. ${ }^{[99]}$

Taking advantage of LM ability to easily roll on a solid surface, new systems may be developed to perform cellular culture in simulated microgravity conditions. Since combining the marbles with a simple lab rotator shaker, LM with cells inside can roll in random directions and maybe replicate the same conditions of the complex systems currently used for simulated microgravity experiments, such as Random Positioning Machine or Rotating Wall Vessels.

\subsubsection{Ultraviolet and Infrared Radiation}

Similarly to the $\mathrm{pH}$-responsive coatings reviewed earlier, the wettability of some special LM coatings can be changed by optical irradiation, without any physical contact. Using photoresponsive material as the coating material, it was reported that the LM rupture can be remotely controllable by using ultraviolet (UV) radiation - Figure 5A. ${ }^{30,100,101]}$ Smart LM were created using a coating of particles that simultaneously were magnetic and $\mathrm{pH}$-responsive, whose hydrophilicity increases when protonated. ${ }^{[30]}$ A photoacid generator within the coating particles was used and when irradiated with UV light generated high concentration of $\mathrm{H}^{+}$that caused the coating particles to become hydrophilic, breaking the LM. Nakai et al. presented a similar photo-responsive LM, but using spiropyran powder to coat water droplets. ${ }^{[100]}$ With UV exposure an isomerization process occurred on the coating material, increasing the coating hydrophilicity. These authors reported to obtain LM that were stable for more than a week in dark and humid conditions, but when exposed to UV radiation the LM burst. Another UV-responsive LM was created by Tan et al., by using hydrophobized titanium 

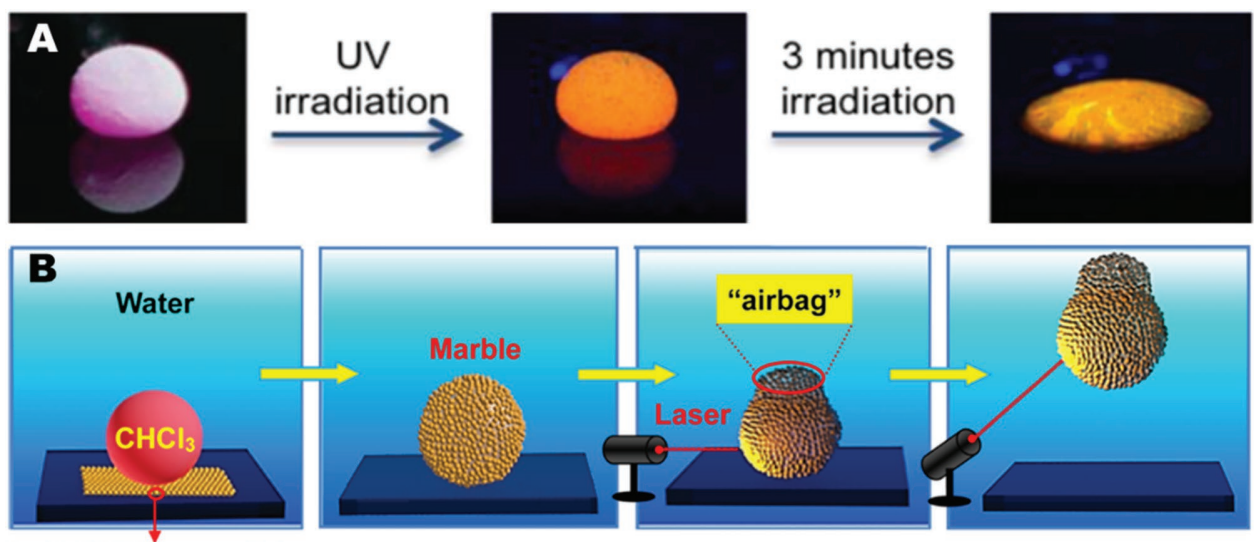

$\mathrm{Fe}_{3} \mathrm{O}_{4} @ P D A$ nanoparticles

Figure 5. A) LM exposed to UV radiation and after several minutes of exposition the LM collapsed. Reproduced with permission. ${ }^{101]}$ Copyright 2014 , American Chemical Society. B) Schematic illustration of remote manipulation of a chloroform $\left(\mathrm{CHCl}_{3}\right)$ marble in water using a near-infrared laser. Reproduced with permission. ${ }^{[104]}$ Copyright 2016, American Chemical Society.

dioxide as the coating particles - Figure 5A. ${ }^{[101]}$ In this case with UV light, hydrophilic hydroxyl groups were formed on coating surface, due to the well-studied photoresponsive behavior of titanium dioxide.

Other way to perform the remote control of LM is by using near-infrared (NIR) laser- Figure 5B. ${ }^{[102-104]}$ One example is the marbles coated with carbon nanotubes and fullerene, which present high absorbance in the NIR region. Upon NIR irradiation, the LM immediately disintegrated due to the transformation of NIR energy into heat, triggering of the bursting of marbles. ${ }^{[102]}$ However, Paven et al. developed a more advanced system that allowed to drive floating LM using simply a NIR laser or sunlight. ${ }^{[103]}$ Comparing with other external stimuli namely $\mathrm{pH}$ and temperature, the light-driven transportation of LM showed advantages such as the control of the timing, position, area, direction and velocity. All of them are important parameters in applications such as the precise delivery and release of materials, which were suggested by the authors. ${ }^{[103]}$ Moreover, the developed system works in a temperature range suitable for biomedical applications $\left(<40{ }^{\circ} \mathrm{C}\right)$, but the biocompatibility of the employed materials with photothermal properties needs to be assessed. Chu et al. also reported to have high control manipulating LM with a NIR laser, but using chloroform marbles submerged in water - Figure $5 B \cdot{ }^{[104]}$ Due to the conversion of NIR energy into heat, vapor bubbles were formed inside of the LM. Controlling the laser irradiation on the marble surface, LM were able to ascend, horizontally move and suspend in water. Authors suggested the applicability of this system for constructing smart micro-reactors, micro-engines or micro-robots in aqueous environment with potential applications in chemical reactions and biological analysis. ${ }^{[104]}$ However, this system is not compatible with cellular culture, due to the toxicity of chloroform which is a key compound of this technology reported by $\mathrm{Chu}$ et al.

\subsubsection{Self-Propulsion}

Taking advantage of LM ability to float, a method was proposed to create self-propelling marbles. ${ }^{[42,60]} \mathrm{LM}$ containing aqueous ethanol solutions were placed on the water surface and they revealed autonomous motion. This motion is explained by the Marangoni solutocapillary effect that happen due to the ethanol evaporation and posterior condensation on the water surface. The ethanol diffusion onto the supporting water generates a gradient of surface tension, impelling motion to the floating object. $^{[42,60]}$ It was found that the lifetime of self-propulsion increased with LM volume and ethanol concentration, ${ }^{[60]}$ but the motion velocity revealed to be independent of the marble volume. ${ }^{[42]}$

This self-propulsion method using ethanol seems not adjustable for biomedical applications. However can be inspiring for the development of a suitable system.

\section{Biomedical Applications}

Due to their peculiar properties and versatility in the design, LM are very promising candidates to be valuable in the biomedical field. Very recently, this new direction on LM applications started to be explored, such as demonstrated by some reports that can be found in literature. ${ }^{[48,61,79,85,105-110]}$ These few works reported very distinctive applications, namely diagnostic assays, cell culture, drug screening and cryopreservation of mammalian cells.

\subsection{Diagnostic Assays}

Due to their small dimensions, the use of LM as miniature reactors has awakened high interest for the miniaturization of chemical processes. Such micro-reactors offer significant advantages namely in reducing the use of chemical reagents and solvents, providing a well-confined micro-environment and a versatile and cost-effective platform. Taking benefit from these LM characteristics, several potential applications have been successfully exploited for chemiluminescence reactions, acid-base reactions, ${ }^{[14]}$ nanocomposite synthesis, ${ }^{[16]}$ polymerizations, ${ }^{[111]}$ silver mirror reactions, ${ }^{[15]}$ and heterogeneous catalytic reactions. ${ }^{[18]}$ An obvious use of LM in the biological area 
is also the miniaturization of processes for biological reactions and diagnostic assays.

The use of LM as micro-bioreactors for rapid blood typing was demonstrated - Figure 6. ${ }^{[105]}$ The "blood marbles" were produced by rolling blood droplets over hydrophobic powder of precipitated calcium carbonate. Sets of three marbles were prepared for each single test, one marble for each one of the three antibody solutions (Anti-A, Anti-B and Anti-D). After injecting on the respective LM the antibody solution, the ABO and $\mathrm{Rh}$ blood grouping was determined by monitoring the occurrence or not of a haemagglutination reaction. The occurrence of this reaction resulted in a two phase separation inside of the marbles, finding clearly a light-red and a dark-red color zone. The dark zone was consequence of a precipitation of the agglutinated red blood cells to the bottom of the LM, indicating the presence of the respective antigen. In contrast, the detection of no separation indicated the absence of the corresponding antigen. From the combined analysis of the results obtained for each one of the three antibodies, the blood grouping was determined - Figure 6B. Blood type assessment is a mandatory step before a blood transfusion to avoid the fatal consequences of incompatibility. Besides the low-cost and disposability of the technique, the authors also claimed that the test can be performed without any special medical facilities and with reduced biohazard due to the encapsulation of the blood samples. ${ }^{[05]}$

Zhao et al. also reported the applicability of LM as suitable platforms for biological assays. ${ }^{[85]}$ They established a new method for the quantitative detection of dopamine in LM based on electrochemical measurements. The authors proposed that this technique can be useful on the diagnosis of neurological disorders in brain functions. ${ }^{[85]}$ Besides this application, a glucose assay based on a colorimetric method was reported. Using magnetic particles for the marble shell production, authors took advantage of the ability to open the magnetic LM with a magnet to perform both measurements. The dopamine detection was performed by immersing a miniaturized electrode in the liquid of the partially opened marble. For the glucose assay, the measure was achieved through the optical absorbance of the liquid in the fully opened LM by transmission mode. Beyond the measurement methods, an integrated approach was proposed for the LM production and analysis using potentially a single device, introduced briefly before - Figure 4B. ${ }^{[85]}$ The magnetic actuation system was envisioned as the way to manipulate (move, open and close) the LM along all of the integrated processes. Complementarily to the electrochemical detection and the transmission-mode optical detection, it was proposed to integrate on the device a reflection-mode optical detection and an encapsulation process performed by infra-red heat-induced. Using wax-based particles in LM preparation, a uniform film surrounding the LM content could be formed by heating. For instance, this encapsulation step allows the samples preservation after analysis for follow-up assessment or biobanking and archiving. ${ }^{[85]}$

\subsection{Cell Culture}

A well-known and explored property of LM are the gas permeable nature of their shell, demonstrate in several

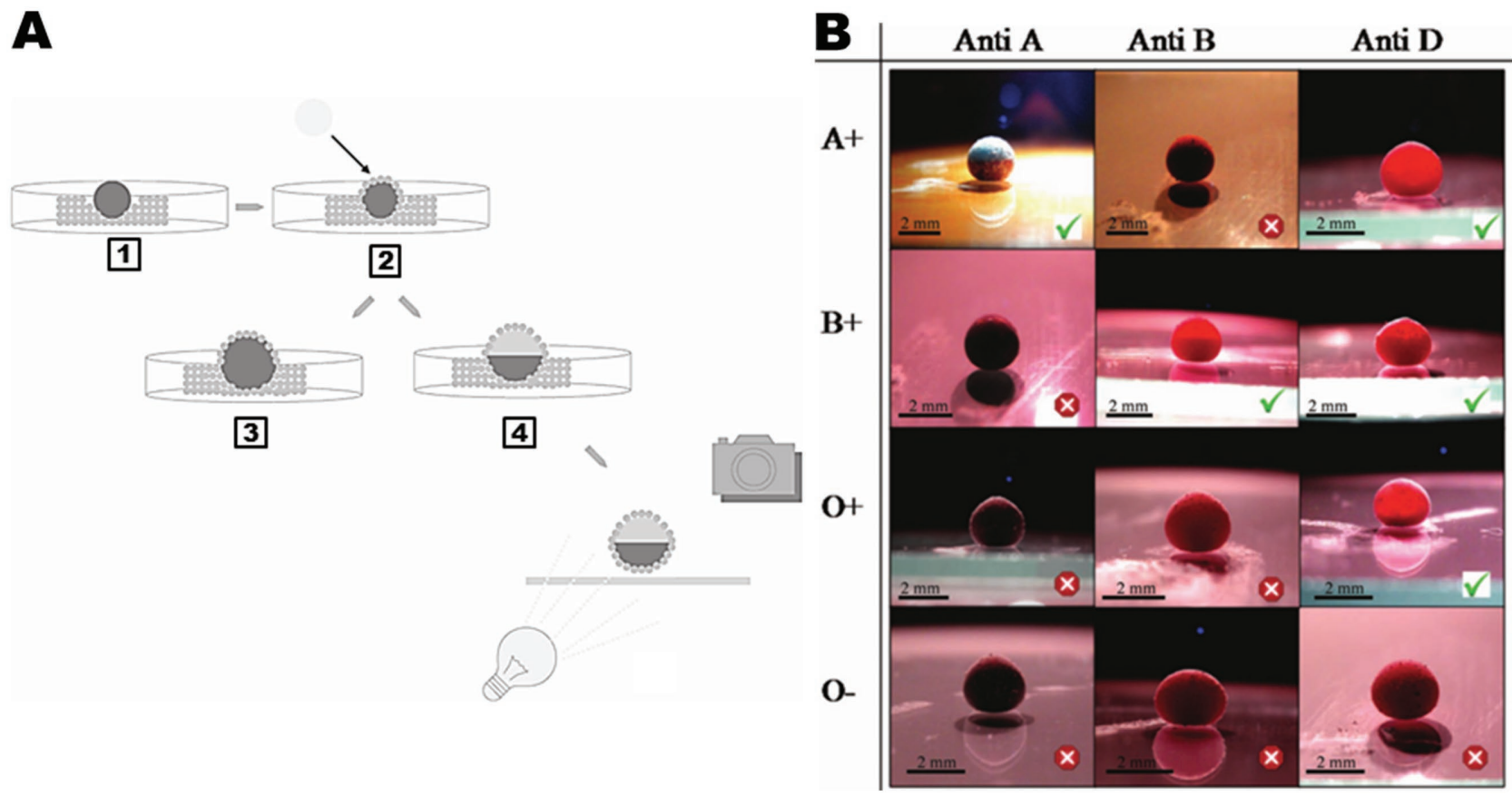

Figure 6. A) Schematic diagram of the stages of micro-bioreactor preparation and blood-type identification. 1) Blood (10 $\mu \mathrm{L})$ is placed on a hydrophobic powder bed to generate the blood marble. 2) An antibody solution ( $10 \mu \mathrm{L})$ is injected inside the blood marble to complete the micro-bioreactor preparation. 3) When the corresponding antigens are not present on the surface of red blood cells, no separation is observable. 4) When the corresponding antigens are present, the agglutination reaction of red blood cells takes place; resulting in the separation of color into two distinct light (top) and dark (bottom) parts. B) Overview of blood typing results after the corresponding antibodies are injected into the micro-bioreactor (20 $\mu \mathrm{L})$. Reproduced with permission. ${ }^{105]}$ 
reports related with gas sensing and gas reactions. ${ }^{[12,13,38-40,111]}$ The gas permeability is a vital feature for LM application in cell culture, allowing the oxygen and carbon dioxide exchange between cell culture medium and surrounding environment. Tian and coworkers demonstrated that LM provided a suitable environment for cell culture by culturing aerobic microorganisms. ${ }^{[41]}$ Besides this work, other 3D bioreactors based on LM have been reported, namely for formations of cancer cell spheroids (CCS), ${ }^{[48]}$ embryoid bodies (EB), ${ }^{[106,107]}$ olfactory ensheathing cell spheroids $^{[61]}$ and for in vitro maturation of sheep oocytes. ${ }^{[108]}$

CCS cultured in vitro are postulated as capable to mimic the in vivo physiology of tumors more realistically than 2D cell cultures. ${ }^{[112-114]}$ These cell spheroids were produced by simply inoculating hepatocellular carcinoma cells in LM. ${ }^{[48]}$ The necessary cell aggregation for CCS formation occurred due to intrinsic characteristics of LM: the confined volume that promoted a higher intercellular interaction; and their non-adhesive shell that discouraged cell adhesion - Figure 7A.

Following the same rationale, the use of these 3D bioreactors for EB formation was also reported, since pluripotent embryonic stem cells tend to form aggregates Figure 7B. ${ }^{[106]}$ LM are presented as a facile and highly efficient means for in vitro production of EB. Additionally to EB formation, it was reported that the LM offered a suitable micro-environment to induce spontaneously (without using growth factors) the EB differentiation into functional cardiomyocytes. ${ }^{[107]}$ The authors emphasized that this technology would be highly beneficial to provide a continuous source of cardiomyocytes for regenerative medicine applications, drug discovery and safety testing. ${ }^{[107]}$

Floating LM may be employed to produce spheroids of olfactory ensheathing cells. ${ }^{[61]}$ The choice for these LM was justified by the fact that floating LM allowed better handling and minimized the effect of evaporation, since the LM were placed over a liquid bath that increased the humidity. Furthermore using floating LM, the effect of gravity that forces cell aggregation at the LM bottom was minimized, due to the motion of the marble over the surface of the supporting liquid improving the mixing process inside the LM. This movement revealed to be crucial to obtain identical size spheroids. In complementary work, it was shown that LM can also be used for co-culture trials. ${ }^{[61]}$ Olfactory ensheathing cells were co-cultured with Schwann cells or astrocytes cells. On the liquid environment provided by LM, the olfactory ensheathing cells were able to replicate their normal behavior of surrounding other cells, because the cells could freely associate with the other cell types. ${ }^{[61]}$

Recently, LM were presented as suitable micro-bioreactors to induce the maturation of oocytes in vitro. ${ }^{[108]}$ Furthermore, it was suggested that LM have high potential for other applications in reproductive biology, such as in oocyte fertilization and individual embryo culture. The reduced reagent consumption and low potential for contamination of LM have been highlighted as valuable characteristics. ${ }^{[108]}$

The use of LM was presented as a valuable alternative to the hanging drop method that is the most common technique used for these reviewed applications. ${ }^{[48,61,106-108]}$

\subsection{Drug Screening}

Taking advantage from the fact that LM allow the injection and extraction of controlled volumes of liquid without disrupting their structure, Oliveira et al. showed that LM could be used for high-throughput drug screening - Figure 8A. ${ }^{[79]}$ Simultaneously, the liquefied environment in the LM was designed to accommodate the culture of anchorage-dependent cells that require a physical support to adhere and be able to proliferate. To accomplish this goal, microparticles were introduced in the 

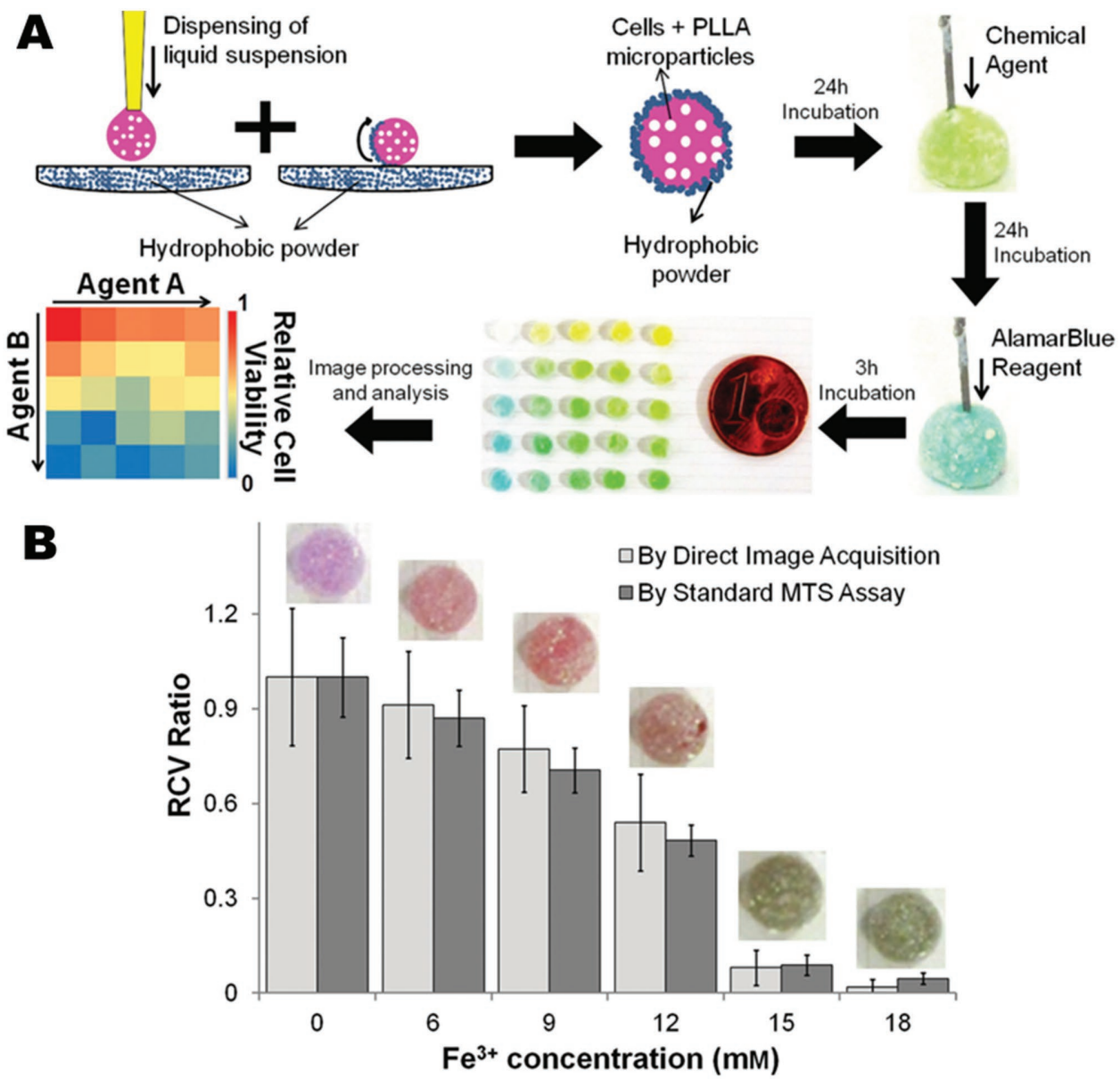

Figure 8. A) Schematic illustration of the method for high-throughput drug screening. B) Relationship between relative cell viability (RCV) ratio and $\mathrm{Fe}^{3+}$ concentration determined using two different methods. A non-destructive method based on image processing is compared with a conventional destructive MTS assay. A characteristic image of LM is shown for each condition. No significant differences were found between the two methods for the studied range of concentrations. Reproduced with permission. ${ }^{[79]}$

liquid environment, providing the indispensable cell anchorage sites for cell adhesion. ${ }^{[79]}$ After preparing the LM with a mixture of cells and microparticles followed by an incubation period for cell adaptation, a drug/chemical agent was injected on the produced micro-bioreactors. Through the injection of a reagent that the color change against a specific cellular response, several conditions could be analyzed and compared. Due to the fact that the LM were translucent, ${ }^{[79]}$ monitoring the color change inside of the LM was possible. This color monitoring was performed by collecting images from LM, and then the final results were obtained by image processing - Figure 8A. The reproducibility of this new technique was fully validated by comparison with a well-known conventional method - Figure 8B. Such technology would have impact in high-throughput drug screening under 3D cell culture environments, where results can be obtained through colorimetric-based and non-destructive measurements. ${ }^{[79]}$

During the assays, the volume/diameter of LM can change due to evaporation, injection and extraction of liquid. However, procedures to mitigate this issue can be easily employed, such as creating an environment of saturated water vapor or managing the injection/extraction procedures in way that ensures a constant volume of the LM. Using non-harmful biochemical reagents for cells such as AlamarBlue, cells wash is not required after biochemical reactions. Respectively, Oliveira et al. and Zhao et al. showed the easy to use of the LM for drug/ biological screening applications - Figure 8 - and for "on-line" electrochemical/optical detection - Figure 9. ${ }^{[79,85]}$ Based on these reports, LM present high potential for high-throughput applications. However, several advances are required on the automatization of processes, since currently all procedures are based on manual manipulation.

\subsection{Cryopreservation of Mammalian Cells}

One very interesting and promising application for LM is their use in cryopreservation of mammalian cells - Figure 10. Serrano et al. presented LM as a successful alternative tool to the conventional methods for the cryopreservation of a mammalian cell line, without using any cryopreservant agents. ${ }^{[09]}$ Most of these agents cause cytotoxic effects requiring to be use at low concentrations, namely dimethyl sulfoxide and glycerol. ${ }^{[115,116]}$ Two procedures inspired on the conventional protocols were 

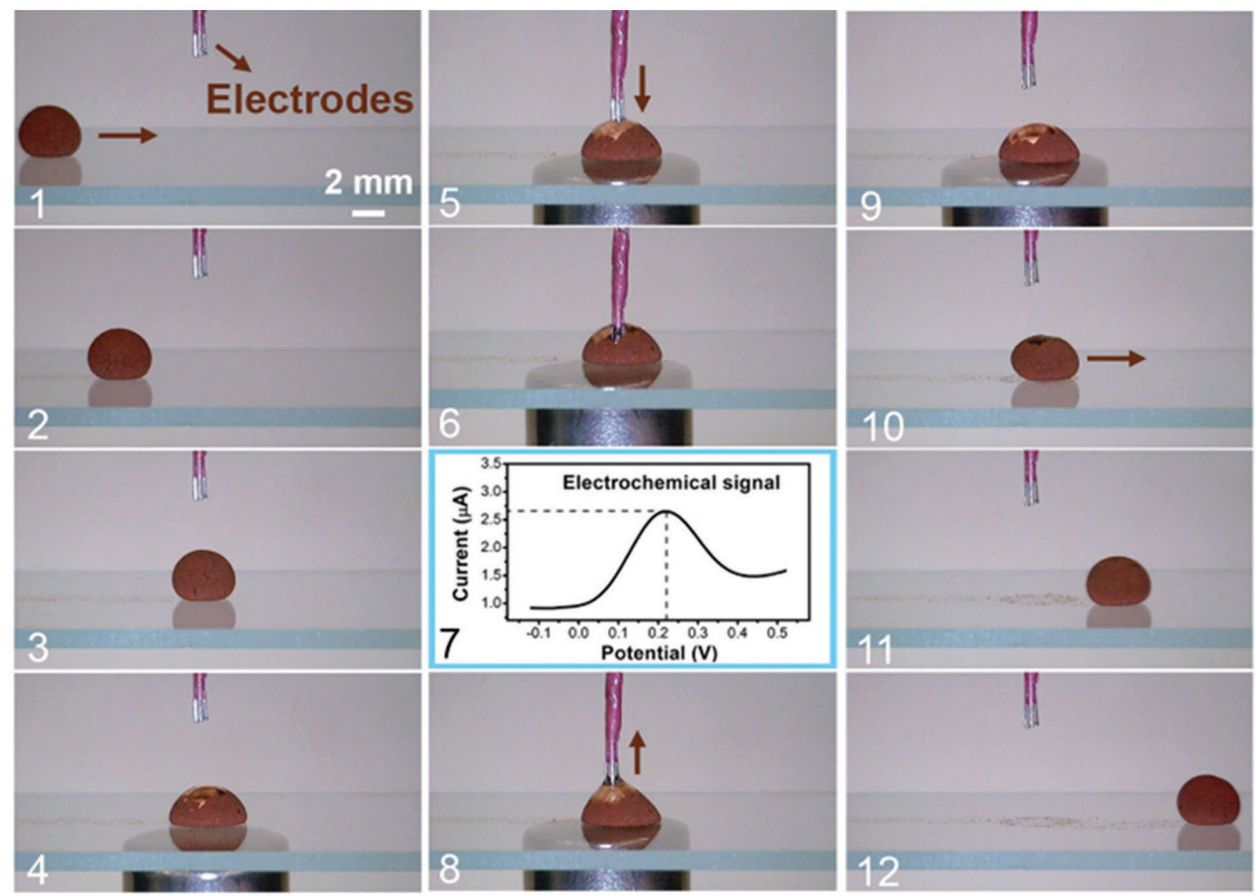

Figure 9. Electrochemical detection: Transport (1-3) of a magnetic liquid marble on glass substrate and its opening (4) with a magnet; Moving threeelectrode probe into the droplet (5-6), recording signal (7), and moving probe out of the droplet (8); Closing (9-10) and moving away (11-12) LM after detection. Reproduced with permission. ${ }^{[85]}$

used: the rapid freezing by immersion in liquid nitrogen and slow cooling up to $-80{ }^{\circ} \mathrm{C}$ using a standard freezing container. LM were prepared by rolling droplets of highly concentrated cell suspension (prepared on fetal bovine serum) over poly(tetrafluoroethylene) powder. ${ }^{[109]}$ Then LM were maintained in frozen conditions for 15 days. After the thawing procedure, the integrity of the LM was confirmed to be intact and the following cellular parameters were evaluated: viability, morphology, proliferation, size, complexity, and cell cycle Figure 10. Droplet volume and cell concentration showed to be the critical factors for using the LM successfully on this application. The authors found a safe range of work: $5-30 \mu \mathrm{L}$ for the droplet volume and $(0.5-2) \times 10^{5}$ cells $\mu \mathrm{L}^{-1}$ for cell concentration. Accomplishing these limits, LM showed to reach the same level of performance of standard freezing procedures for all the evaluated cellular parameters. ${ }^{[109]}$

\section{Critical Analysis: The Potential of Liquid Marbles for Biomedical Applications}

Due to their peculiar properties and versatility in the design, LM are very promising candidates to be valuable in the biomedical field. The LM present a high versatility such as shown in the several applications already reported in literature, mainly on the chemistry field. Some of these applications can be just adapted or may inspire new applications for biomedical purposes. The majority of the reported data concerning evaporation, floatability and robustness of LM was obtained using water as the encapsulated liquid. Equivalent studies should be performed using cell culture medium and human body fluids, because the surface tension for these fluids is different from water. ${ }^{[105,117,118]}$ Being the surface tension of the encapsulated liquid one of the crucial parameters that influence LM behavior, ${ }^{[2,3]}$ specific studies are required to find the appropriated materials to encapsulate these fluids.

LM present high potential for application in the development of co-culture models to mimic in vivo environments, because they can ensure a compartmentalized 3D cell culture environment. ${ }^{[112-114]}$ This application for the LM started to be explored by Vadivelu et al., where they studied the interaction of olfactory ensheathing cells with Schwann cells and with astrocytes. They reported that olfactory ensheathing cells wrapped the other cells, behavior that was observed for the first time. ${ }^{[61]}$ We believe that other co-culture models can be created using the same method by mixing different cell types in a single LM. Alternatively, we also envisage that in the first stage different 3D cell structures (spheroids or cell/microparticle aggregates) can be produced in separated LM. Then, by the coalescence of two or more marbles into a larger one, the produced 3D structures can be co-culture in the same confined environment. ${ }^{[98,99]}$ Other opportunity is to perform indirect co-culture of cells, using a connecting tube between two LM with different cell types in culture and study the paracrine signaling on cellular response. A similar scheme was reported by Bormashenko et al., where they connected two different LM with a capillary tube to build a micro-pump powered by the differences on the surface tension between two different marbles. ${ }^{[19]}$

Other more complex and revolutionary co-culture scheme can be to produce capsules with cells inside generated from LM. Specifically the idea is that LM spontaneously become capsules through a chemical or physical process that promotes 


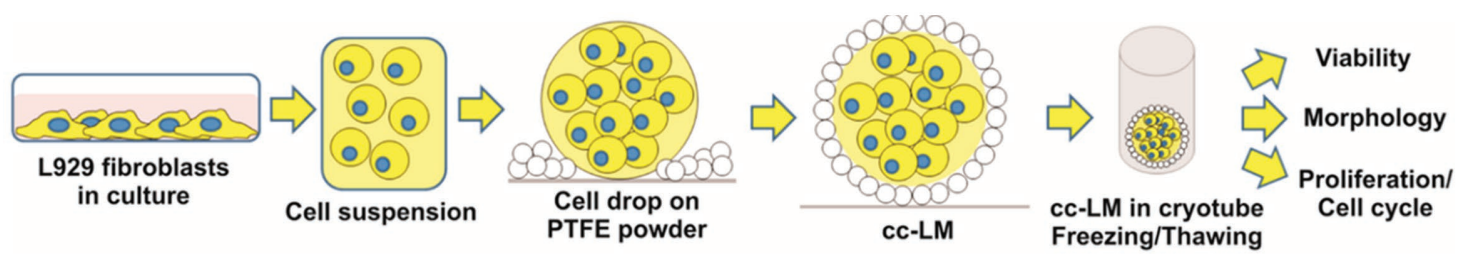

Figure 10. Scheme of the developed procedure for cryopreservation of mammalian cells. Reproduced with permission. ${ }^{[109]}$ Copyright 2015 , American Chemical Society.

the merging between the coating particles creating a membrane. ${ }^{[15,18,119]}$ Incubating different cell types on these capsules, a kind of "organoid" structures can be produced and the biochemical communication between them can be studied, ensuring that the capsule membrane is permeable to both nutrients and cellular metabolites. Such kind of compartmentalization of cellular microenvironments were performed before using the layer-by-layer technology. ${ }^{[120,121]}$ "Organoids" produced with LM could be then cultured together in the same culture well or integrated in a fluidic system, following the same rationale of the "organ-on-a-chip". ${ }^{[22,123]}$ Chin et al. already reported a process to produce stable liquid capsules from LM, promoting an interfacial polymerization by vaporization of ethyl-2cyanoacrylate. ${ }^{[119]}$ Other works showed that the coating particles of LM can be part of chemical reactions happing inside of the marbles, performing a role as catalytic particles ${ }^{[18]}$ or reactive substrates. ${ }^{[15]}$ However, these solutions are not suitable to be used in cell culture, but we believe that similar processes can be found by combining different biocompatible materials.

Having in mind the suggestion of Zhao et al. for the integration of several processes in a single device and using the magnetism for LM manipulation, ${ }^{[85]}$ a similar device can be developed for biomedical applications. We propose the development of an advanced high-throughput system for cytotoxic analysis of drugs and materials. The system may incorporate the several steps for this kind of assessment: production of magnetic LM with cells; incubation of cells for the formation of single cell spheroids or 3D cell/microparticle aggregates or co-culture spheroids (see Figure 11); ${ }^{48,61,79,106,107]}$ optical morphology assessment of the 3D cell structures; drug/ material supply by injection or direct access to the LM core; ${ }^{[14,48,78,79]}$ cellular response assessment by colorimetric methods or electrochemical measurements; ${ }^{[79,85]}$ by on-line analysis of results, the sorting of LM can be performed; then using the selected LM, the cells can be maintained in culture on the same platform or moved for other environment by LM burst; cells from the selected conditions can also be used in more powerful methods for cellular evaluation such as flow cytometry. ${ }^{[109]}$ We believe that almost all of these processes can be fully automatized on the proposed device. Besides the application in high-throughput analysis, such proposed platform could also find application in diagnostic assays.
Here, the suggested systems allow to create 3D cellular structures in biomaterial-free environments or almost free, without the requirement of using scaffolds to obtain 3D organization. 3D spheroids of living cells are of high interest as models for basic science, playing a crucial role in cancer research, drug discovery and tissue engineering. ${ }^{[124-126]}$ The growing significance assumed by spheroids on biomedical research is justified by their similarity to the native $3 \mathrm{D}$ biological functions present in in vivo tissues. In 3D cellular structures, cell-to-cell interactions are amplified, better mimicking all functional and architectural features of native tissues, as compared to 2D cell culture. ${ }^{[114]}$

Inspired by the LM, Oliveira et al. created a novel concept termed "hydrophobic hydrogels", consisting into coat hydrogels with hydrophobic particles. ${ }^{[110]}$ They found that similarly to LM the created hydrogels could float. Based on this ability, floating (bio)microdevices were developed for different applications, namely optical $\mathrm{pH}$ sensing, microengineering of selfassembling floating hydrogels and construction of engineered biological tissues. ${ }^{[110]}$ A step forward on this technology can be the use of these floating hydrogels to create in vitro skin tissue, once this tissue prefer the air-liquid interface. Possibly, a stratified structure similar to skin tissue can be obtained by encapsulating cells on the hydrogel and other cell type cultured on the hydrogel top at the air-liquid interface. To culture cells on the hydrogel top, the surface should be free of hydrophobic particles and can be achieved by controlling the covered hydrogel surfaces in the coating process. Moreover, to ensure
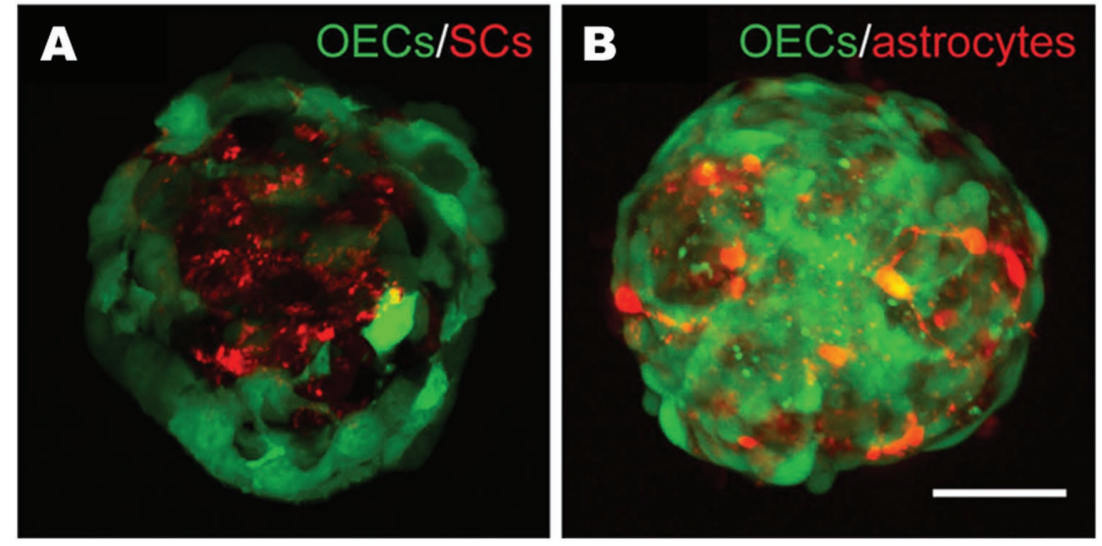

Figure 11. Co-culture of olfactory ensheathing cells (OECs) with Schwann cells and with astrocytes. Marbles were seeded with the two different cell types at a ratio of 1:1 with an overall seeding density of 500 cells $/ \mu \mathrm{L}$ and cultured for $48 \mathrm{~h}$. Spheroid co-cultures of (A) OECs (green) with Schwann cells (red) and (B) OECs (green) with astrocytes (red). Scale bar: $60 \mu \mathrm{m}$. Reproduced with permission. ${ }^{[61]}$ Copyright 2015, Nature Publishing Group. 
that the air-liquid interface remains at the same position (above the liquid surface) with the same exposed area, the produced hydrogel should have the shape of a cube or a parallelepiped. Thus, the bottom surface of the cube/parallelepiped will ensure the nutrients exchange with the surrounding liquid where the hydrogel is floating. The top surface will be in constant contact with air and the lateral surfaces should be coated by the hydrophobic particles to guarantee the hydrogel stability without rotate through its horizontal axis.

Based on the published knowledge about LM, we believe that several applications on the biomedical field can be reported soon. Some applications seem more straightforward to reach, such as using LM as a tool for cryopreservation of particularly sensitive cells, namely pluripotent stem cells. ${ }^{[109,127]}$ Other applications should be more difficult to reach, such as some of the suggested applications presented here in this section.

\section{Summary}

This review deals with the recent advanced on LM field and the emerging applications on the biomedical area, showing the natural evolution of some applications from other scientific fields to the biomedical engineering.

The interfacial characteristics of LM were largely explained, namely the shell structure that present primordial influence on the LM properties. This relationship between the coating and the LM capabilities was described, specifically for the capability of LM to deform and float. The evaporation phenomenon and robustness were elucidated, because are features with high importance for long term applications such as occasionally required on the biological and biomedical fields. The manipulation of LM is also a critical issue on this field to avoid contaminations. However, several methods have being reported for LM handling without direct actuation over the marbles. The remote manipulation can be performed by magnetic, electrostatic or gravitational forces and even by using UV or NIR light.

The biomedical applications found for the LM were reviewed, in a perspective how the LM properties and manipulation methods were explored for this aim. Applications on diagnosis, drug screening, cell culture and cryopreservation were described. Finalizing, a critical view of the LM potential for biomedical applications was presented, suggesting some possible advances on this emerging area.

\section{Acknowledgements}

The authors acknowledge funding from the European Research Council grant agreement ERC-2012-ADC 20120216-321266 for project ComplexiTE. N. M. Oliveira acknowledges the financial support from Portuguese Foundation for Science and Technology - FCT (Grant SFRH/ $\mathrm{BD} / 73172 / 2010)$, from the financial program POPH/FSE from QREN.

\section{Conflict of Interest}

The authors declare no conflict of interest.

\section{Keywords}

biomedical applications, freestanding droplets, liquid marbles, soft solids

Received: February 9, 2017

Revised: May 20, 2017

Published online:

[1] P. Aussillous, D. Quere, Nature 2001, 411, 924.

2] P. Aussillous, D. Quere, Proc. R. Soc., A 2006, 462, 973.

[3] E. Bormashenko, Curr. Opin. Colloid Interface Sci. 2011, 16, 266.

[4] G. McHale, M. I. Newton, Soft Matter 2015, 11, 2530.

[5] M. Dandan, H. Y. Erbil, Langmuir 2009, 25, 8362.

[6] E. Bormashenko, R. Pogreb, A. Musin, R. Balter, G. Whyman, D. Aurbach, Powder Technol. 2010, 203, 529.

[7] B. Wang, Y. Liu, Y. B. Zhang, Z. G. Guo, H. Zhang, J. H. Xin, L. Zhang, Adv. Mater. Interfaces 2015, 2, 1500234.

[8] S. Y. Huang, Y. Zhang, J. F. Shi, W. P. Huang, ACS Sustain. Chem. Eng. 2016, 4, 676.

[9] J. H. Sun, W. Wei, D. H. Zhao, Q. Hu, X. Y. Liu, Soft Matter 2015, 11, 1954.

[10] K. Ueno, G. Bournival, E. J. Wanless, S. Nakayama, E. C. Giakoumatos, Y. Nakamura, S. Fujii, Soft Matter 2015, 11, 7728.

[11] S. Ohno, Y. Tsuda, K. Nakai, S. Fujii, Y. Nakamura, S. Yusai, Chem. Lett. 2016, 45, 547.

[12] J. F. Tian, T. Arbatan, X. Li, W. Shen, Chem. Commun. 2010, 46, 4734.

[13] S. Fujii, K. Aono, M. Suzaki, S. Hamasaki, S. Yusa, Y. Nakamura, Macromolecules 2012, 45, 2863.

[14] Y. H. Xue, H. X. Wang, Y. Zhao, L. M. Dai, L. F. Feng, X. G. Wang, T. Lin, Adv. Mater. 2010, 22, 4814.

[15] Y. F. Sheng, G. Q. Sun, J. Wu, G. H. Ma, T. Ngai, Angew. Chem. Int Ed. 2015, 54, 7012.

[16] Y. Chu, Z. K. Wang, Q. M. Pan, ACS Appl. Mater. Interfaces 2014, 6, 8378.

[17] E. Bormashenko, A. Musin, Appl. Surf. Sci. 2009, 255, 6429

[18] W. Wei, R. J. Lu, W. T. Ye, J. H. Sun, Y. Zhu, J. Luo, X. Y. Liu, Langmuir 2016, 32, 1707.

[19] E. Bormashenko, R. Balter, D. Aurbach, Appl. Phys. Lett. 2010, 97, 091908.

[20] S. Yue, W. Shen, K. Hapgood, Adv. Powder Technol. 2016, 27, 33.

[21] G. McHale, M. I. Newton, Soft Matter 2011, 7, 5473.

[22] E. Bormashenko, Soft Matter 2012, 8, 11018.

[23] H. Y. Erbil, Adv. Colloid Interfaces 2012, 170, 67.

[24] C. H. Ooi, N. T. Nguyen, Microfluid Nanofluid 2015, 19, 483.

[25] S. Nagappan, C. S. Ha, J. Mater. Chem. A 2015, 3, 3224.

[26] T. L. Sun, L. Feng, X. F. Gao, L. Jiang, Acc. Chem. Res. 2005, 38 644.

[27] H. Y. Erbil, A. L. Demirel, Y. Avci, O. Mert, Science 2003, 299, 1377.

[28] P. Roach, N. J. Shirtcliffe, M. I. Newton, Soft Matter 2008, 4, 224.

[29] Y. Zhao, J. Fang, H. X. Wang, X. G. Wang, T. Lin, Adv. Mater. 2010, 22, 707.

[30] L. B. Zhang, D. K. Cha, P. Wang, Adv. Mater. 2012, 24, 4756.

[31] A. M. S. Costa, N. V. Dencheva, S. G. Caridade, Z. Z. Denchev, J. F. Mano, Adv. Mater. Interfaces 2016, 3, 1600074.

[32] T. H. Nguyen, K. Hapgood, W. Shen, Chem. En. J. 2010, 162, 396.

[33] A. M. Fernandes, D. Mantione, R. Gracia, J. R. Leiza, M. Paulis, D. Mecerreyes, ACS Appl. Mater. Interfaces 2015, 7, 4433.

[34] E. Bormashenko, Y. Bormashenko, A. Musin, Z. Barkay, ChemPhysChem 2009, 10, 654. 
[35] D. Matsukuma, H. Watanabe, A. Fujimoto, K. Uesugi, A. Takeuchi, Y. Suzuki, H. Jinnai, A. Takahara, Bull. Chem. Soc. Jpn. 2015, 88, 84.

[36] W. Barthlott, C. Neinhuis, Planta 1997, 202, 1.

[37] U. Cengiz, H. Y. Erbil, Soft Matter 2013, 9, 8980.

[38] J. F. Tian, T. Arbatan, X. Li, W. Shen, Chem. Eng. J. 2010, 165, 347.

[39] M. M. Hu, M. J. Tian, J. He, Y. J. He, Colloid Surf. A 2012, 414, 216.

[40] K. Matsubara, M. Danno, M. Inoue, H. Nishizawa, Y. Honda, T. Abe, Surf. Coat. Technol. 2013, 236, 269.

[41] J. F. Tian, N. Fu, X. D. Chen, W. Shen, Colloid Surf. B 2013, 106, 187.

[42] E. Bormashenko, Y. Bormashenko, R. Grynyov, H. Aharoni, G. Whyman, B. P. Binks, J. Phys. Chem. C 2015, 119, 9910.

[43] L. Mahadevan, Y. Pomeau, Phys. Fluids 1999, 11, 2449.

[44] D. Richard, D. Quere, Europhys. Lett. 1999, 48, 286.

[45] C. H. Ooi, R. K. Vadivelu, J. St John, D. V. Dao, N. T. Nguyen, Soft Matter 2015, 11, 4576.

[46] D. Vella, D. G. Lee, H. Y. Kim, Langmuir 2006, 22, 5979.

[47] G. Whyman, E. Bormashenko, J. Colloid Interface Sci. 2009, 331, 174.

[48] T. Arbatan, A. Al-Abboodi, F. Sarvi, P. P. Y. Chan, W. Shen, Adv. Healthcare Mater. 2012, 1, 467.

[49] G. McHale, N. J. Shirtcliffe, M. I. Newton, F. B. Pyatt, S. H. Doerr, Appl. Phys. Lett. 2007, 90, 054110.

[50] A. Tosun, H. Y. Erbil, Appl. Surf. Sci. 2009, 256, 1278.

[51] P. S. Bhosale, M. V. Panchagnula, H. A. Stretz, Appl. Phys. Lett. 2008, 93, 034109

[52] P. S. Bhosale, M. V. Panchagnula, Langmuir 2010, 26, 10745

[53] B. Laborie, F. Lachaussee, E. Lorenceau, F. Rouyer, Soft Matter 2013, 9, 4822.

[54] S. Ogawa, H. Watanabe, L. M. Wang, H. Jinnai, T. J. McCarthy, A. Takahara, Langmuir 2014, 30, 9071.

[55] C. H. Ooi, E. Bormashenko, A. V. Nguyen, G. M. Evans, D. V. Dao, N. T. Nguyen, Langmuir 2016, 32, 6097.

[56] L. C. Gao, T. J. McCarthy, Langmuir 2007, 23, 10445.

[57] D. Dupin, S. P. Armes, S. Fujii, J. Am. Chem. Soc. 2009, 131, 5386.

[58] S. Fujii, S. Kameyama, S. P. Armes, D. Dupin, M. Suzaki, Y. Nakamura, Soft Matter 2010, 6, 635.

[59] D. Dupin, K. L. Thompson, S. P. Armes, Soft Matter 2011, 7, 6797.

[60] C. H. Ooi, A. V. Nguyen, G. M. Evans, O. Gendelman, E. Bormashenko, N. T. Nguyen, Rsc Adv. 2015, 5, 101006.

[61] R. K. Vadivelu, C. H. Ooi, R. Q. Yao, J. T. Velasquez, E. Pastrana, J. Diaz-Nido, F. Lim, J. A. K. Ekberg, N. T. Nguyen, J. A. St John, Sci. Rep. 2015, 5, 15085.

[62] C. H. Ooi, C. Plackowski, A. V. Nguyen, R. K. Vadivelu, J. A. John, D. V. Dao, N. T. Nguyen, Sci. Rep. 2016, 6, 21777.

[63] Y. F. Si, Z. G. Guo, Chem. Lett. 2016, 45, 779.

[64] K. Nakai, S. Fujii, Y. Nakamura, S. Yusa, Chem. Lett. 2015, 44, 1077.

[65] G. Whyman, E. Bormashenko, J. Colloid Interface Sci. 2015, 457, 148.

[66] S. Asare-Asher, J. N. Connor, R. Sedev, J. Colloid Interface Sci. 2015, 449, 341.

[67] E. Bormashenko, Langmuir 2017, 33, 663.

[68] E. Bormashenko, G. Whyman, O. Gendelman, Adv. Cond. Matter Phys. 2015, 2015, 206578.

[69] E. Bormashenko, R. Pogreb, R. Balter, H. Aharoni, Y. Bormashenko, R. Grynyov, L. Mashkevych, D. Aurbach, O. Gendelman, Colloid Polym. Sci. 2015, 293, 2157.

[70] L. Rayleigh, Proc. R. Soc. Lond. 1879, 29, 71.

[71] D. Richard, C. Clanet, D. Quere, Nature 2002, 417, 811.

[72] Z. Liu, X. Y. Fu, B. P. Binks, H. C. Shum, Langmuir 2015, 31, 11236.

[73] D. Y. Zang, E. Rio, G. Delon, D. Langevin, B. Wei, B. P. Binks, Mol. Phys. 2011, 109, 1057.

[74] D. Y. Zang, Z. Chen, Y. J. Zhang, K. J. Lin, X. G. Geng, B. P. Binks, Soft Matter 2013, 9, 5067.
[75] T. Kostakis, R. Ettelaie, B. S. Murray, Langmuir 2006, 22, 1273.

[76] X. M. Zhou, X. X. Lin, K. L. White, S. Lin, H. Wu, S. L. Cao, L. L. Huang, L. H. Chen, Cellulose 2016, 23, 811.

[77] L. Forny, I. Pezron, K. Saleh, P. Guigon, L. Komunjer, Powder Technol. 2007, 171, 15.

[78] A. Bajwa, Y. H. Xu, A. Hashmi, M. Leong, L. Ho, J. Xu, Soft Matter 2012, 8, 11604

[79] N. M. Oliveira, C. R. Correia, R. L. Reis, J. F. Mano, Adv. Healthcare Mater. 2015, 4, 264.

[80] G. Q. Sun, Y. F. Sheng, J. Wu, G. H. Ma, T. Ngai, Langmuir 2014, 30, 12503.

[81] E. Bormashenko, R. Pogreb, Y. Bormashenko, A. Musin, T. Stein, Langmuir 2008, 24, 12119.

[82] N. T. Nguyen, Microfluid Nanofluid 2012, 12, 1.

[83] Y. Zhao, Z. G. Xu, M. Parhizkar, J. Fang, X. G. Wang, T. Lin, Microfluid Nanofluid 2012, 13, 555.

[84] F. G. Li, W. Wu, A. F. Ning, J. Wang, Int. J. Chem. React. Eng. 2015, $13,113$.

[85] Y. Zhao, Z. G. Xu, H. T. Niu, X. G. Wang, T. Lin, Adv. Funct. Mater. $2015,25,437$.

[86] M. K. Khaw, C. H. Ooi, F. Mohd-Yasin, R. Vadivelu, J. St John, N. T. Nguyen, Lab Chip 2016, 16, 2211.

[87] L. X. Li, B. C. Li, L. Fan, B. Mu, A. Q. Wang, J. P. Zhang, J Mater Chem A 2016, 4, 5859

[88] X. X. Lin, W. Ma, H. Wu, S. L. Cao, L. L. Huang, L. H. Chen, A. Takahara, Chem. Commun. 2016, 52, 1895.

[89] J. Sun, S. B. Zhou, P. Hou, Y. Yang, J. Weng, X. H. Li, M. Y. Li, J. Biomed. Mater. Res. A 2007, 80A, 333.

[90] M. I. Newton, D. L. Herbertson, S. J. Elliott, N. J. Shirtcliffe, G. McHale, J. Phys. D, Appl. Phys. 2007, 40, 20.

[91] G. McHale, D. L. Herbertson, S. J. Elliott, N. J. Shirtcliffe, M. I. Newton, Langmuir 2007, 23, 918.

[92] K. R. Liyanaarachchi, P. M. Ireland, G. B. Webber, K. P. Galvin, Appl. Phys. Lett. 2013, 103, 054105.

[93] E. Bormashenko, R. Pogreb, T. Stein, G. Whyman, M. Schiffer, D. Aurbach, J. Adhes. Sci. Technol. 2011, 25, 1371.

[94] E. Bormashenko, R. Pogreb, R. Balter, O. Gendelman, D. Aurbach, Appl. Phys. Lett. 2012, 100, 151601.

[95] E. Bormashenko, R. Pogreb, R. Balter, H. Aharoni, D. Aurbach, V. Strelnikov, Petrol. Sci. 2015, 12, 340.

[96] E. Bormashenko, Y. Bormashenko, R. Pogreb, O. Gendelman, Langmuir 2011, 27, 7.

[97] P. Aussillous, D. Quere, J. Fluid Mech. 2004, 512, 133.

[98] C. Planchette, A. L. Biance, O. Pitois, E. Lorenceau, Phys. Fluids 2013, 25, 042104.

[99] J. O. Castro, B. M. Neves, A. R. Rezk, N. Eshtiaghi, L. Y. Yeo, ACS Appl. Mater. Interfaces 2016, 8, 17751.

[100] K. Nakai, S. Fujii, Y. Nakamura, S. Yusa, Chem. Lett. 2013, 42, 586.

[101] T. T. Y. Tan, A. Ahsan, M. R. Reithofer, S. W. Tay, S. Y. Tan, T. S. A. Hor, J. M. Chin, B. K. J. Chew, X. B. Wang, Langmuir 2014, 30,3448 .

[102] K. Nakai, H. Nakagawa, K. Kuroda, S. Fujii, Y. Nakamura, S. Yusa, Chem. Lett. 2013, 42, 719.

[103] M. Paven, H. Mayama, T. Sekido, H. J. Butt, Y. Nakamura, S. Fujii, Adv. Funct. Mater. 2016, 26, 3199.

[104] Y. Chu, F. T. Liu, L. M. Qin, Q. M. Pan, ACS Appl. Mater. Interfaces 2016, 8, 1273.

[105] T. Arbatan, L. Z. Li, J. F. Tian, W. Shen, Adv. Healthcare Mater. 2012, 1,80

[106] F. Sarvi, T. Arbatan, P. P. Y. Chan, W. Shen, Rsc Adv. 2013, 3, 14501.

[107] F. Sarvi, K. Jain, T. Arbatan, P. J. Verma, K. Hourigan, M. C. Thompson, W. Shen, P. P. Y. Chan, Adv. Healthcare Mater. $2015,4,77$

[108] S. Ledda, A. Idda, J. Kelly, F. Ariu, L. Bogliolo, D. Bebbere, J. Assist. Reprod. Gen. 2016, 33, 513. 
[109] M. C. Serrano, S. Nardecchia, M. C. Gutierrez, M. L. Ferrer, F. del Monte, ACS Appl. Mater. Interfaces 2015, 7, 3854

[110] N. M. Oliveira, Y. S. Zhang, J. Ju, A. Z. Chen, Y. Chen, S. R. Sonkusale, M. R. Dokmeci, R. L. Reis, J. F. Mano, A. Khademhosseini, Chem. Mater. 2016, 28, 3641 .

[111] E. Sato, M. Yuri, S. Fujii, T. Nishiyama, Y. Nakamura, H. Horibe, Chem. Commun. 2015, 51, 17241.

[112] W. MuellerKlieser, Am. J. Physiol., Cell Physiol. 1997, 273, C1109.

[113] K. M. Yamada, E. Cukierman, Cell 2007, 130, 601.

[114] F. Pampaloni, E. G. Reynaud, E. H. K. Stelzer, Nat. Rev. Mol. Cell Biol. 2007, 8, 839.

[115] R. J. Klebe, M. G. Mancuso, In Vitro Cell Dev. B 1983, 19, 167.

[116] M. S. Kim, S. T. Lee, J. M. Lim, S. P. Gong, Cytotechnology 2016, $68,9$.

[117] N. B. Vargaftik, B. N. Volkov, L. D. Voljak, J. Phys. Chem. Ref. Data $1983,12,817$.

[118] J. Rosina, E. Kvasnak, D. Suta, H. Kolarova, J. Malek, L. Krajci, Physiol. Res. 2007, 56, S93.
[119] J. M. Chin, M. R. Reithofer, T. T. Y. Tan, A. G. Menon, E. Y. Chen, C. A. Chow, A. T. S. Hor, J. W. Xu, Chem. Commun. 2013, 49, 493.

[120] C. R. Correia, R. P. Pirraco, M. T. Cerqueira, A. P. Marques, R. L. Reis, J. F. Mano, Sci. Rep. 2016, 6, 21883.

[121] C. R. Correia, S. Gil, R. L. Reis, J. F. Mano, Adv. Healthcare Mater. 2016, 5, 1346

[122] C. Moraes, G. Mehta, S. C. Lesher-Perez, S. Takayama, Ann. Biomed. Eng. 2012, 40, 1211

[123] F. Y. Zheng, F. F. Fu, Y. Cheng, C. Y. Wang, Y. J. Zhao, Z. Z. Gu, Small 2016, 12, 2253

[124] T. M. Achilli, J. Meyer, J. R. Morgan, Expert Opin. Biol. Th. 2012, 12, 1347.

[125] J. M. Kelm, E. Ehler, L. K. Nielsen, S. Schlatter, J. C. Perriard, M. Fussenegger, Tissue Eng. 2004, 10, 201.

[126] V. Mironov, V. Kasyanov, R. R. Markwald, Curr. Opin. Biotech. 2011, 22, 667.

[127] R. I. Cohen, M. L. Thompson, B. Schryver, R. O. Ehrhardt, in Current Protocols in Stem Cell Biology 2014, https://doi. org/10.1002/9780470151808.sc01c14s28. 